\title{
Erratum: Quantum Error Correction of Photon Loss with Quantum Encoding
}

\author{
[J. Korean Phys. Soc. 62, 721 (2013)]
}

Chao HAN*

Faculty of Science, Ningbo University, Ningbo 315211, China

DOI: $10.3938 / \mathrm{jkps} .64 .1087$

The notation " " in the second line of section III should be replaced by " $\Sigma$ ".

The paragraph from equation (2) to (4) should be corrected as follow:

Assuming that the first photon is lost, the density matrix of the system is traced over this qubit and results in an equal mixture of two pure state projections onto the even and odd bit-string parity sector of the Hilbert space of the remaining qubits, which is

$$
\begin{aligned}
\rho_{0}^{\prime}= & \left\{\left(c_{1}|0000000\rangle+c_{2}|0000011\rangle+c_{3}|0001100\rangle+c_{4}|0001111\rangle\right.\right. \\
& \left.+c_{5}|0110000\rangle+c_{6}|0110011\rangle+c_{7}|0111100\rangle+c_{8}|0111111\rangle, \frac{1}{2}\right), \\
& +\left(c_{1}|1111111\rangle+c_{2}|1111100\rangle+c_{3}|1110011\rangle+c_{4}|1110000\rangle\right. \\
& \left.\left.+c_{5}|1001111\rangle+c_{6}|1001100\rangle+c_{7}|1000011\rangle+c_{8}|1000000\rangle, \frac{1}{2}\right)\right\} .
\end{aligned}
$$

Detecting the error by the QND device, the subsequent single-photon gun (SPG) prepares a photon in ground state to replace the lost photon. The mixed state then becomes

$$
\begin{aligned}
\rho_{1}= & \left\{\left(c_{1}|00000000\rangle+c_{2}|00000011\rangle+c_{3}|00001100\rangle+c_{4}|00001111\rangle\right.\right. \\
& \left.+c_{5}|00110000\rangle+c_{6}|00110011\rangle+c_{7}|00111100\rangle+c_{8}|00111111\rangle, \frac{1}{2}\right), \\
& +\left(c_{1}|01111111\rangle+c_{2}|01111100\rangle+c_{3}|01110011\rangle+c_{4}|01110000\rangle\right. \\
& \left.\left.+c_{5}|01001111\rangle+c_{6}|01001100\rangle+c_{7}|01000011\rangle+c_{8}|01000000\rangle, \frac{1}{2}\right)\right\} .
\end{aligned}
$$

